\title{
Approximate Schur Preconditioners for Efficient Solutions of Dielectric Problems Formulated with Surface Integral Equations
}

\author{
Tahir Malas ${ }^{1,2}$ and Levent Gürel ${ }^{1,2}$ \\ ${ }^{1}$ Department of Electrical and Electronics Engineering \\ ${ }^{2}$ Computational Electromagnetics Research Center (BiLCEM) \\ Bilkent University, TR-06800, Bilkent, Ankara, Turkey \\ \{tmalas,lgurel\}@ee.bilkent.edu.tr
}

\begin{abstract}
We propose direct and iterative versions of approximate Schur preconditioners to increase robustness and efficiency of iterative solutions of dielectric problems formulated with surface integral equations. The performance of these preconditioners depends on the availability of fast and approximate solutions to reduced matrix systems. We show that sparse-approximateinverse techniques provide a suitable mechanism for this purpose. The proposed preconditioners are demonstrated to significantly improve convergence rates of dielectric problems formulated with two different surface integral equations.
\end{abstract}

\section{INTRODUCTION}

Discretization of surface integral-equation formulations of dielectric problems leads to dense, complex, and nonhermitian linear systems. Those linear systems have an explicit $2 \times 2$ partitioned structure in the form of

$$
\left[\begin{array}{ll}
\overline{\boldsymbol{A}}_{11} & \overline{\boldsymbol{A}}_{12} \\
\overline{\boldsymbol{A}}_{21} & \overline{\boldsymbol{A}}_{22}
\end{array}\right] \cdot\left[\begin{array}{c}
\boldsymbol{v}_{J} \\
\boldsymbol{v}_{M}
\end{array}\right]=\left[\begin{array}{l}
\boldsymbol{b}_{1} \\
\boldsymbol{b}_{2}
\end{array}\right] \text { or } \overline{\boldsymbol{A}} \cdot \boldsymbol{v}=\boldsymbol{b},
$$

where $\overline{\boldsymbol{A}} \in \mathbb{C}^{2 N \times 2 N}, \overline{\boldsymbol{A}}_{11}, \overline{\boldsymbol{A}}_{12}, \overline{\boldsymbol{A}}_{21}$, and $\overline{\boldsymbol{A}}_{22} \in \mathbb{C}^{N \times N}, \boldsymbol{v}_{J}$ and $\boldsymbol{v}_{M}$ are $N \times 1$ coefficient vectors of the Rao-WiltonGlisson [1] basis functions expanding the equivalent electric and magnetic electric currents, respectively. In (1), $\boldsymbol{b}_{1}$ and $\boldsymbol{b}_{2}$ represent $N \times 1$ excitation vectors obtained by testing the incident electric and magnetic fields. Since the system matrices in (1) are dense, it is customary to use iterative methods for the solutions and to employ the multilevel fast multipole algorithm (MLFMA) [2] to accelerate the dense matrix-vector multiplications (MVMs).

We consider preconditioning of linear systems that arise from the combined tangential formulation (CTF) and the electric and magnetic current combined-field integral equation (JMCFIE), which is a combination of CTF and the combined normal formulation (CNF) [3]-[5]. Being a first-kind integral equation, $\mathrm{CTF}$ produces more accurate results than other integral-equation formulations, but solving the resulting linear system is difficult without effective preconditioning. Similar to the case in perfect-electric-conductor (PEC) problems, being a combined formulation, JMCFIE is very efficient for solving large-scale problems [6]. Accuracy of the solutions obtained via JMCFIE, however, can be significantly poorer than those of CTF [7]. Moreover, when the dielectric constant of the problem increases, JMCFIE matrices tend to be less wellconditioned. Hence, effective preconditioning is indispensable for accurate and efficient solutions of dielectric problems.

To provide a fast MVM, MLFMA decomposes the dense coefficient matrix as $\overline{\boldsymbol{A}}=\overline{\boldsymbol{A}}^{N F}+\overline{\boldsymbol{A}}^{F F}$, where $\overline{\boldsymbol{A}}^{F F}$ denotes the far-field matrix and $\overline{\boldsymbol{A}}^{N F}$ the near-field matrix. Only $\overline{\boldsymbol{A}}^{N F}$ is stored in the memory, hence, we use this sparse matrix as the preconditioning matrix (i.e., the matrix from which the preconditioner is to be constructed). Then, preconditioners are obtained with some approximations to the Schur complement reduction [8], which decouples the solution of a partitioned linear system to the solutions of reduced systems with the $(1,1)$ partition and the Schur complement. We show how to solve these systems approximately and efficiently by employing sparse-approximate-inverse (SAI) techniques [9].

\section{Surface Integral Equations for Dielectric PROBLEMS}

Recently, significant progress has been made in devising new integral-equation formulations that are suitable for iterative solutions of dielectric problems [3]-[5]. Among these formulations, we will briefly review CTF, which produces the most accurate results, and JMCFIE, which requires smallest iteration counts for large problem sizes [6].

\section{A. $C T F$}

Surface formulations that are free of internal-resonance problems can be obtained by combining integral equations of the outer and the inner regions of the object. For example, CTF [3] is defined by combining inner (I) and outer $(\mathrm{O})$ versions of the tangential electric-field integral equation (T-EFIE) and the tangential magnetic-field integral equation (T-MFIE), i.e.,

$$
\begin{aligned}
& \frac{1}{\eta_{1}} \text { T-EFIE-O }+\frac{1}{\eta_{2}} \text { T-EFIE-I, } \\
& \eta_{1} \text { T-MFIE-O }+\eta_{2} \text { T-MFIE-I, }
\end{aligned}
$$

where $\eta_{1}$ and $\eta_{2}$ are the impedances of the outer and inner regions, respectively. In (2), the identity terms of inner and outer integral equations cancel each other, and CTF turns out 
to be a first-kind integral equation that has a smooth kernel [7]. The smoothing property of the kernel results in coefficient matrices that are far from being diagonally dominant and that have poor conditioning. On the other hand, due to this smoothing property of its kernel, CTF has a better solution accuracy compared to normal formulations, such as CNF.

\section{B. JMCFIE}

By using a normal electric-field integral equation (N-EFIE) and a normal magnetic-field integral equation (N-MFIE), second-kind normal formulations can be obtained, such as CNF [3]. Even though the singular kernels and the identity terms of normal formulations lead to more diagonally dominant matrices and better conditioning than CTF, accuracy of such formulations can be much worse than that of CTF.

Compared to CNF, JMCFIE is a more accurate second-kind integral-equation formulation, which is obtained by combining $\mathrm{CTF}$ and $\mathrm{CNF}$ as [5]

$$
\mathrm{JMCFIE}=\alpha \mathrm{CTF}+\beta \mathrm{CNF},
$$

where $0 \leq \alpha \leq 1$ and $\beta=1-\alpha$. In addition to being more accurate than CNF, the matrix systems of JMCFIE formulation are more stable and can be solved usually in fewer iterations, compared to those of CNF [6].

However, both the solution accuracy and the conditioning of JMCFIE decrease as the dielectric constant increases [7]. When object surfaces have sharp edges and corners, they also have negative effects on the accuracy of JMCFIE. Therefore, when the dielectric constant is high and/or the surface of the object has non-smooth sections, the accuracy of JMCFIE can be much poorer than the accuracy of CTF [7]. Therefore, preconditioning is a critical issue for accurate and efficient electromagnetics simulations of dielectric objects. If, in some cases, the accuracy of the normal formulations and JMCFIE are unacceptable, then one may have to employ CTF, for which the solutions are tough to obtain without effective preconditioning. For high dielectric constants, JMCFIE tends to produce matrices that are not well-conditioned, necessitating the application of effective preconditioners.

\section{ASP}

For the solution of the preconditioning system

$$
\left[\begin{array}{ll}
\overline{\boldsymbol{A}}_{11}^{N F} & \overline{\boldsymbol{A}}_{12}^{N F} \\
\overline{\boldsymbol{A}}_{21}^{N F} & \overline{\boldsymbol{A}}_{22}^{N F}
\end{array}\right] \cdot\left[\begin{array}{l}
\boldsymbol{x} \\
\boldsymbol{y}
\end{array}\right]=\left[\begin{array}{l}
\boldsymbol{f} \\
\boldsymbol{g}
\end{array}\right],
$$

we use the method of Schur complement reduction that reduces the solution of (4) to the solution of the following two systems. First, $\boldsymbol{y}$ is found using

$$
\overline{\boldsymbol{S}} \cdot \boldsymbol{y}=\boldsymbol{g}-\overline{\boldsymbol{A}}_{21}^{N F} \cdot\left(\overline{\boldsymbol{A}}_{11}^{N F}\right)^{-1} \cdot \boldsymbol{f},
$$

where

$$
\overline{\boldsymbol{S}}=\overline{\boldsymbol{A}}_{22}^{N F}-\overline{\boldsymbol{A}}_{21}^{N F} \cdot\left(\overline{\boldsymbol{A}}_{11}^{N F}\right)^{-1} \cdot \overline{\boldsymbol{A}}_{12}^{N F}
$$

is the Schur complement matrix. Then, $\boldsymbol{x}$ can be computed by solving

$$
\overline{\boldsymbol{A}}_{11}^{N F} \cdot \boldsymbol{x}=\boldsymbol{f}-\overline{\boldsymbol{A}}_{12}^{N F} \cdot \boldsymbol{y}
$$

Approximate solutions of (5) and (7) can serve as useful preconditioners. These solutions can be obtained either directly or iteratively, i.e., either by directly approximating the inverses of $\overline{\boldsymbol{A}}_{11}^{N F}$ and $\overline{\boldsymbol{S}}$ or by employing an iterative solver. We call the former "approximate Schur preconditioner (ASP)" and the latter "iterative ASP (IASP)". Note that approximate inverses or incomplete LU (ILU) factors are still required as "inner" preconditioners for efficient IASP implementations. Hence, for both ASP and IASP, the effectiveness of the preconditioner depends on devising fast approximations for the $(1,1)$ partition $\overline{\boldsymbol{A}}_{11}^{N F}$ and the Schur complement $\overline{\boldsymbol{S}}[10]$.

\section{A. Approximating the Inverse of the $(1,1)$ Partition}

Since the exact inversion of the sparse matrix $\overline{\boldsymbol{A}}_{11}^{N F}$ is too expensive, we can use SAIs to approximate the inverse of $\overline{\boldsymbol{A}}_{11}^{N F}$. We use the same pattern of $\overline{\boldsymbol{A}}_{11}^{N F}$ for the approximate inverse, hence, their memory consumptions are the same. Advantages of using SAIs over ILU factors are robustness and ease of parallelization. Furthermore, by using the block structure of the the near-field matrix, we can eliminate the high setup time of SAI.

In Fig. 1, we depict the extreme eigenvalues bounding the spectra for matrices $\overline{\boldsymbol{M}}_{11} \cdot \overline{\boldsymbol{A}}_{11}^{N F}$, where $\overline{\boldsymbol{M}}_{11}$ denotes the SAI of $\overline{\boldsymbol{A}}_{11}^{N F}$. These eigenvalues are obtained with no-restart GMRES [11]. The geometry is a $4 \lambda$-diameter sphere involving 29,742 unknowns. We see that eigenvalues are very tightly clustered around the point $(1,0)$ for JMCFIE. For CTF, we see a slightly looser clustering than JMCFIE. Also note that the spectra of $\overline{\boldsymbol{A}}_{11}^{N F}$ are not significantly affected with the increase of the dielectric constant.
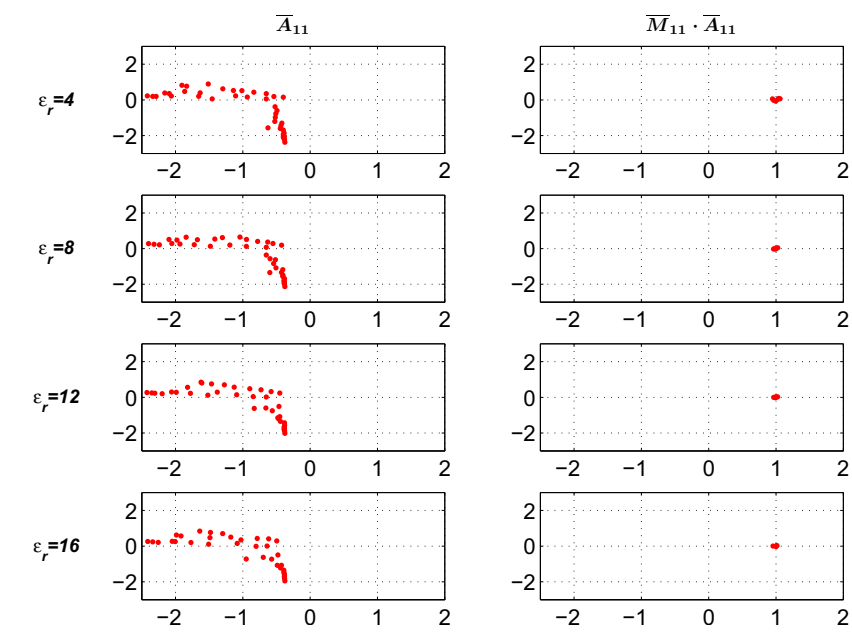

Fig. 1. Eigenvalues of $\overline{\boldsymbol{M}}_{11} \cdot \overline{\boldsymbol{A}}_{11}^{N F}$ for dielectric constants of 4 and 12.

\section{B. Approximating the Inverse of the Schur Complement}

The approximation for the inverse of the Schur complement matrix is more delicate than that of $\overline{\boldsymbol{A}}_{11}^{N F}$. Furthermore, the approximation level provided to the solution of the system involving $\bar{S}$ should be similar to the approximation level provided to the solution of the system involving $\overline{\boldsymbol{A}}_{11}^{N F}[12]$. 
As a first choice, we can approximate the inverse of the Schur complement matrix as

$$
\overline{\boldsymbol{S}}^{-1} \approx\left(\overline{\boldsymbol{A}}_{22}^{N F}\right)^{-1} \approx \overline{\boldsymbol{M}}_{22},
$$

assuming that the first term in the RHS of (6) is the dominant term in the Schur complement. Since $\overline{\boldsymbol{A}}_{22}^{N F}=\overline{\boldsymbol{A}}_{11}^{N F}$ for CTF and JMCFIE, $\overline{\boldsymbol{M}}_{22}=\overline{\boldsymbol{M}}_{11}$, and we can use the same SAI of the $(1,1)$ partition for the Schur complement as well. In Fig. 2, we evaluate this approximation by depicting the boundary eigenvalues of matrices $\overline{\boldsymbol{M}}_{22} \cdot \overline{\boldsymbol{S}}$. We observe that the spectra of JMCFIE are significantly scattered compared to those in Fig. 1 with a high dielectric constant. Similar to JMCFIE, but to a lesser extent, the spectra of CTF are also scattered. However, we can use $\overline{\boldsymbol{M}}_{22}$ as an inner preconditioner of IASP, provided that the dielectric constant of a problem is not very high.
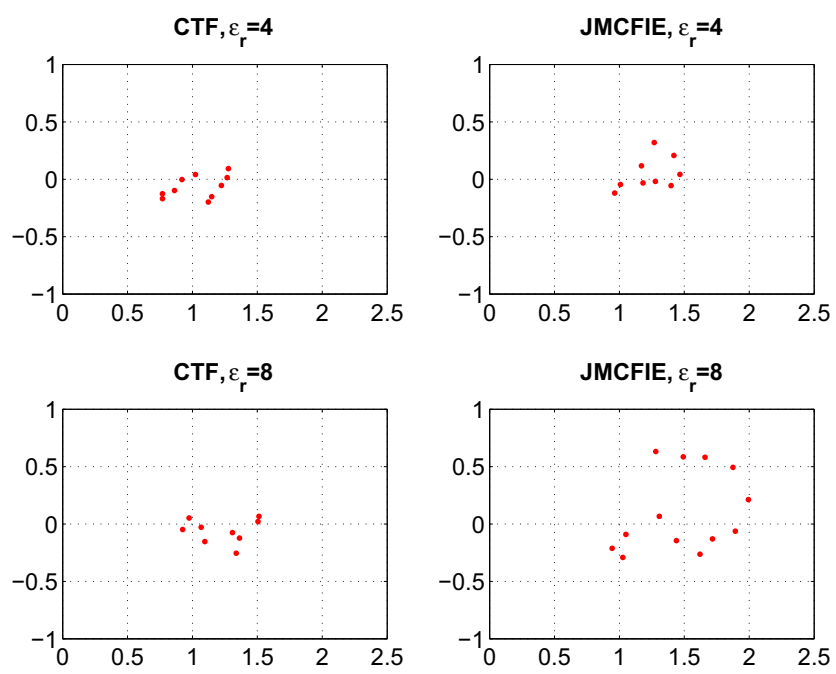

Fig. 2. Eigenvalues of $\overline{\boldsymbol{M}}_{22} \cdot \overline{\boldsymbol{S}}$ for dielectric constants of 4 and 12 .

As another choice, we can generate an explicit SAI for $\bar{S}$ that involves the second term of the Schur complement by employing incomplete matrix-matrix multiplications. First, we compute a sparse approximation to $\bar{S}$ in the form of

$$
\widetilde{\boldsymbol{S}}=\overline{\boldsymbol{A}}_{22}^{N F}-\overline{\boldsymbol{A}}_{21}^{N F} \odot \overline{\boldsymbol{M}}_{11} \odot \overline{\boldsymbol{A}}_{12},
$$

where $\odot$ denotes an incomplete matrix-matrix multiplication obtained by retaining the near-field sparsity pattern. Then, the approximation is performed as

$$
\overline{\boldsymbol{S}}^{-1} \approx \widetilde{\overline{\boldsymbol{S}}}^{-1} \approx \overline{\boldsymbol{M}}_{\text {Schur }},
$$

where $\overline{\boldsymbol{M}}_{\text {Schur }}$ denotes a SAI approximation to the inverse of $\widetilde{\boldsymbol{S}}$. The incomplete matrix-matrix multiplication can be performed in $\mathcal{O}(N)$ time using the $i k j$ loop order of the block matrix-matrix multiplication [13] since the block entries of the near-field partitions are stored row-wise. This operation is detailed with a pseudocode in Fig. 3. Note that the "if" statement in the innermost loop ensures that a block of $\overline{\boldsymbol{C}}_{i j}$ is updated only if clusters $i$ and $j$ are in the near-field of each other. This way, the near-field sparsity pattern is preserved for the product partition $\bar{C}$.

$$
\begin{aligned}
& \overline{\boldsymbol{C}}=\overline{\mathbf{0}} \\
& \text { for each lowest-level cluster } i \text { do } \\
& \text { for each cluster } k \in \mathcal{N}(i) \text { do } \\
& \quad \text { for each cluster } j \in \mathcal{N}(k) \text { do } \\
& \quad \text { if } j \in \mathcal{N}(i) \text { then } \\
& \quad \overline{\boldsymbol{C}}_{i j}=\overline{\boldsymbol{C}}_{i j}+\overline{\boldsymbol{D}}_{i k} \cdot \overline{\boldsymbol{E}}_{k j} \\
& \quad \text { endif } \\
& \quad \text { endfor } \\
& \text { endfor } \\
& \text { endfor }
\end{aligned}
$$

Fig. 3. Incomplete matrix-matrix multiplication of $\overline{\boldsymbol{C}}=\overline{\boldsymbol{D}} \cdot \overline{\boldsymbol{E}}$, where $\overline{\boldsymbol{C}}$ $\overline{\boldsymbol{D}}$, and $\overline{\boldsymbol{E}}$ are block near-field partitions having the same sparsity pattern. $\overline{\boldsymbol{C}}_{i j}$ denotes the block of the near-field partition $\overline{\boldsymbol{C}}$ that corresponds to the interaction of the cluster $i$ with cluster $j . \mathcal{N}(i)$ denotes the clusters that are in the near-field zone of cluster $i$.

We evaluate the approximation (10) in Fig. 4. It is clear that $\overline{\boldsymbol{M}}_{\text {Schur }}$ provides a more successful approximation to the inverse of the Schur complement $\bar{S}$ than $\overline{\boldsymbol{M}}_{22}$. When we compare Figs. 1 and 4 , we observe that the approximation level provided by $\overline{\boldsymbol{M}}_{\text {Schur }}$ is close to that of $\overline{\boldsymbol{M}}_{11}$.
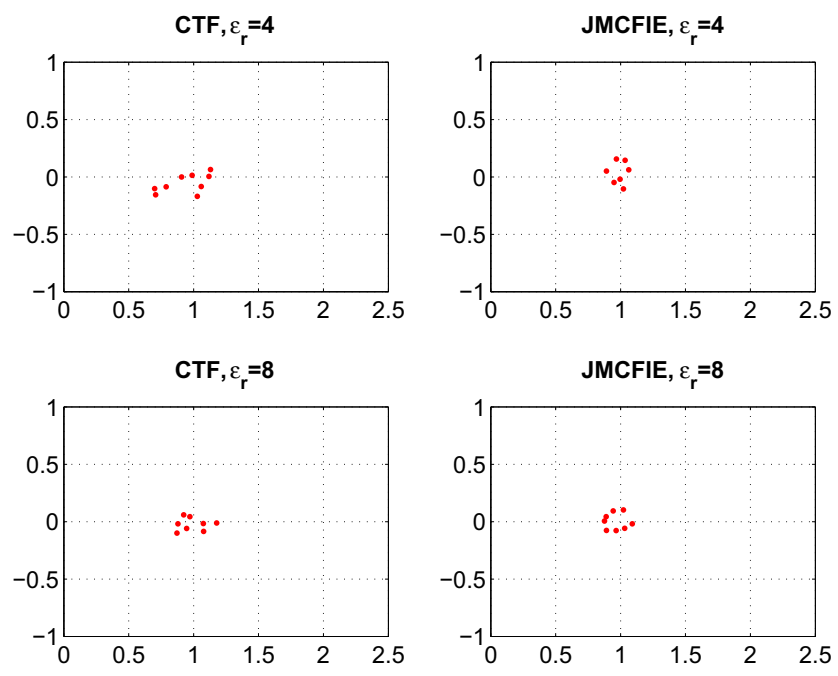

Fig. 4. Eigenvalues of $\overline{\boldsymbol{M}}_{\text {Schur }} \cdot \overline{\boldsymbol{S}}$ for dielectric constants of 4 and 12.

\section{NumericAl RESUlts}

In our experiments, we use the generalized minimal residual method (GMRES) [14] with no restart as the iterative solver. We note that there is a significant difference between the performances of GMRES and other non-optimal solvers, such as biconjugate gradient stabilized, for the solutions of dielectric problems [6]. Iterations are performed until the norm of the initial residual is reduced by a factor of $10^{-3}$. Solutions are started with a zero initial guess and terminated if 1,000 iterations are reached. We use $\alpha=\beta=0.5$ for the JMCFIE formulation.

We compare no-preconditioner (No PC), a four-partition block-diagonal preconditioner (4PBDP) [6], and an ILU-type 
preconditioner (ILU(0) or ILUT [14]) with ASP and IASP. 4PBDP is a simple preconditioner constructed using only the self interactions of the last-level clusters in each partition. For JMCFIE, we select ILU(0) among the ILU-type preconditioners since JMCFIE shows some kind of diagonal dominance. For CTF, we employ the dual-threshold ILUT preconditioner, which has proven to be a robust and effective preconditioner for the PEC case [15]. We set threshold values so that ILUT uses up the same amount of memory with $\operatorname{ILU}(0)$ and the near-field matrix [15]. For IASP, we use $\overline{\boldsymbol{M}}_{11}$ (approximate inverse of the $(1,1)$ partition) as the sole inner preconditioner, hence, the memory requirement of IASP is only one-fourth of that of the ILU-type preconditioners. For ASP, we use $\overline{\boldsymbol{M}}_{\text {Schur }}$ for the Schur complement instead of $\overline{\boldsymbol{M}}_{11}$, hence, the memory requirement of ASP is half of that of the ILU-type preconditioners. In order to provide an indication about the optimality of the preconditioners, we also present the iteration counts for the case where the whole near-field matrix $\overline{\boldsymbol{A}}^{N F}$ is solved exactly by an LU factorization.

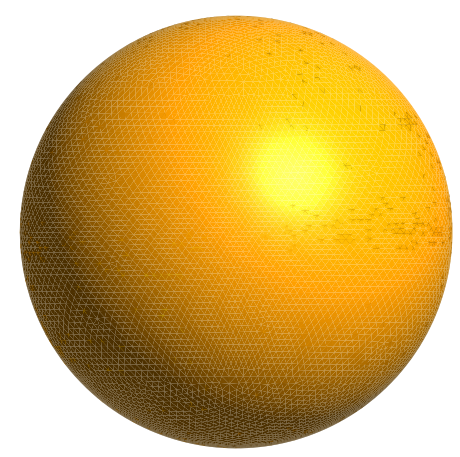

Fig. 5. The sphere problem used in the numerical experiments. The dielectric constant of the problem is 4 .

In Fig. 5, we depict the sphere problem, which has a dielectric constant of 4. In Figs. 6 and 7, we show the experiments carried out with this problem. For CTF, 4PBDP decelerates the convergence rate, since CTF is far from being diagonally dominant. ILUT also do not provide a significant improvement over No PC. A similar observation has been reported before and it was shown that ILUT is also ineffective in a finiteelement implementation of the Navier-Stokes equations [8]. On the other hand, both 4PBDP and ILU(0) significantly improves convergence rates of JMCFIE matrices, compared to No PC. However, both ASP and IASP performs much better than other preconditioners for CTF and JMCFIE. For both formulations, IASP performs significantly better than ASP, and the iteration counts of IASP become very close to those of LU. For IASP, we use a 0.1 residual error and at most three inner iterations, hence, the application cost of this preconditioner is modest. As a result, IASP also significantly improves the solution times compared to ASP.

Then, we comment on the setup time of preconditioners. The algorithm given in Fig. 3 performs incomplete matrixmatrix multiplications very fast, and as a result there is a minor difference between the construction times $\boldsymbol{M}_{11}$ and $\overline{\boldsymbol{M}}_{\text {Schur. }}$. For example, for the large sphere problem that involves 540,450 unknowns, setup time of $\overline{\boldsymbol{M}}_{11}$ is only 5.7 minutes and that of $\overline{\boldsymbol{M}}_{\text {Schur }}$ is 6.2 minutes. As Fig. 7 reveals, these times are negligible compared to the iteration times. ILU(0) also has a negligible setup time. However, ILUT requires substantial setup time when the number of unknowns is large. For the largest sphere problem, for example, setup of ILUT requires 220 minutes.
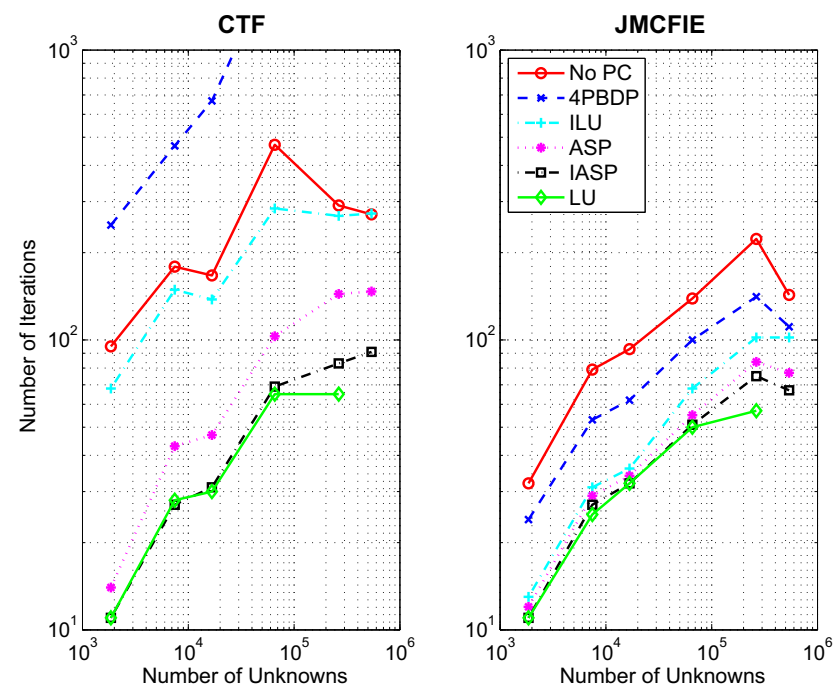

Fig. 6. Number of iterations for the sphere problem obtained with various preconditioners.
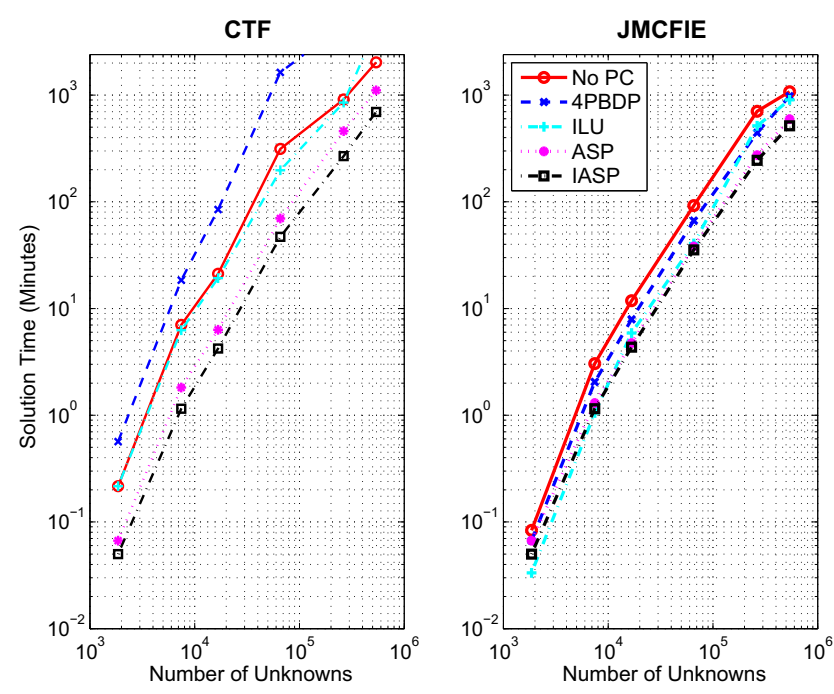

Fig. 7. Total solution times (setup of the preconditioner and iterations) for the sphere problem obtained with various preconditioners.

Next, we consider a lens problem shown in Fig. 8, which has a higher dielectric constant of 12 [16]. We use ASP for this problem since $\overline{\boldsymbol{M}}_{22}$ becomes a poor approximation to the inverse of the Schur complement for high dielectric constants as shown in Fig. 2. In Figs. 9 and 10, we show the iteration 


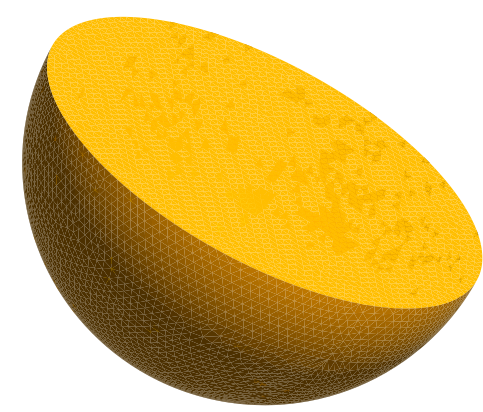

Fig. 8. The lens problem used in the numerical experiments. The dielectric constant of the problem is 12 .

counts and solution times related to a series of problems between $30 \mathrm{GHz}$ and $120 \mathrm{GHz}$, with increasing numbers of unknowns from 38,466 to 632,172 . Performances of 4PBDP and ILU-type preconditioners for this problem are similar to their performances for the sphere problem. However, ASP preconditioner produces iteration counts that are very close to those of LU for this problem, and we do not need to use IASP. For both CTF and JMCFIE, ASP solves the lens problems significantly faster than ILU-type preconditioners and with only half as much memory.
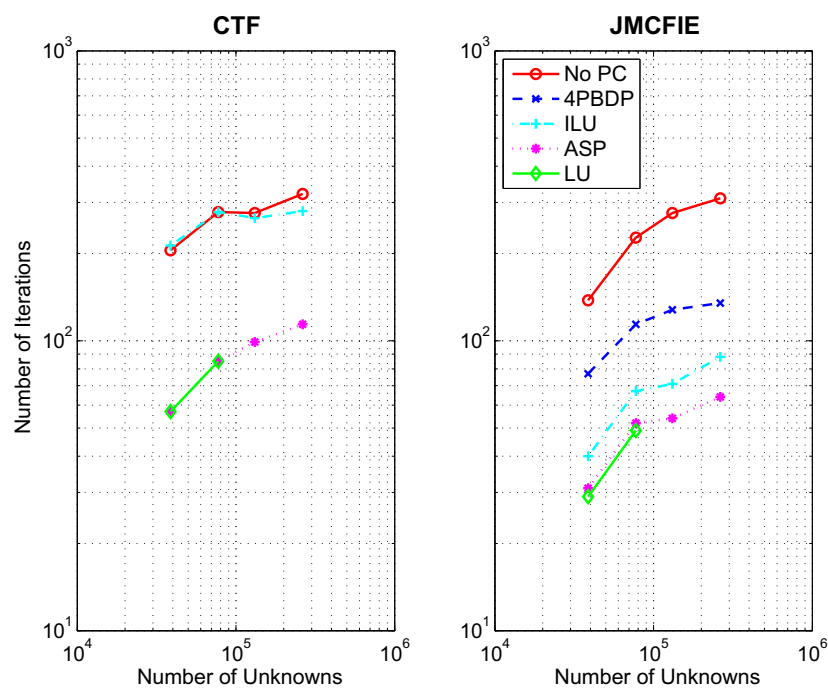

Fig. 9. Number of iterations for the lens problem obtained with various preconditioners.

\section{CONCLUSIONS}

We have developed robust Schur complement preconditioners (ASP and IASP) for dielectric problems formulated with surface integral equations. The success of those preconditioners depends on the approximate and fast computations of the inverses of the near-field $(1,1)$ partition and the Schur complement. For the $(1,1)$ partition, we employ a SAI, which uses the same sparsity pattern of the partition. For the Schur complement, we can use the same SAI, provided that the
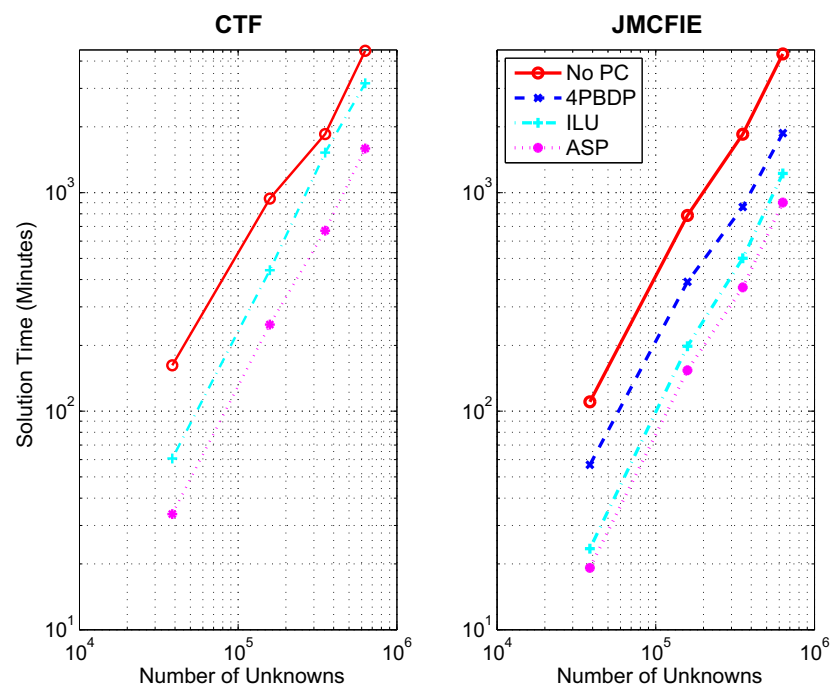

Fig. 10. Total solution times (setup of the preconditioner and iterations) for the lens problem obtained with various preconditioners.

solutions with these systems are found iteratively (IASP) and the dielectric constant of the problem is not very high. This way, we reduce the memory requirement by four fold, compared to ILU-type preconditioners that have the same sparsity ratio as the near-field matrix. For high dielectric constants, however, we need a better approximation for the Schur complement. We showed that this approximation can be obtained via incomplete matrix-matrix multiplications.

The proposed ASP preconditioners have negligible setup times and low memory requirements. They render difficult dielectric problems solvable for both the first-kind CTF and the second-kind JMCFIE formulations.

\section{ACKNOWLEDGMENT}

This work was supported by the Scientific and Technical Research Council of Turkey (TUBITAK) under Research Grants 105E172 and 107E136, by the Turkish Academy of Sciences in the framework of the Young Scientist Award Program (LG/TUBA-GEBIP/2002-1-12), and by contracts from ASELSAN and SSM.

\section{REFERENCES}

[1] S. Rao, D. R. Wilton, and A. W. Glisson, "Electromagnetic scattering by surfaces of arbitrary shape," IEEE Trans. Antennas Propagat., vol. AP-30, pp. 409-418, 1982.

[2] W. C. Chew, J.-M. Jin, E. Michielssen, and J. Song, Eds., Fast and Efficient Algorithms in Computational Electromagnetics. Norwood, MA, USA: Artech House, Inc., 2001.

[3] P. Ylä-Oijala, M. Taskinen, and S. Järvenpää, "Surface integral equation formulations for solving electromagnetic scattering problems with iterative methods," Radio Science, vol. 40, RS6002, doi:10.1029/2004RS003169, no. 6, 2005.

[4] P. Ylä-Oijala and M. Taskinen, "Well-conditioned Muller formulation for electromagnetic scattering by dielectric objects," IEEE Trans. Antennas Propagat., vol. 53, no. 10, pp. 3316-3323, 2005.

[5] _ , "Application of combined field integral equation for electromagnetic scattering by composite metallic and dielectric objects," IEEE Trans. Antennas Propagat., vol. 53, no. 3, pp. 1168-1173, 2005. 
[6] Ö. Ergül and L. Gürel, "Comparison of integral-equation formulations for the fast and accurate solution of scattering problems involving dielectric objects with multilevel fast multipole algorithm," IEEE Trans. Antennas Propagat., vol. 57, pp. 176-187, Jan. 2009.

[7] P. Ylä-Oijala, M. Taskinen, and S. Järvenpää, "Analysis of surface integral equations in electromagnetic scattering and radiation problems," Eng. Anal. Boundary Elem., vol. 32, no. 3, pp. 196-209, 2008.

[8] E. Chow and Y. Saad, "Approximate inverse techniques for blockpartitioned matrices," SIAM J. Sci. Comput., vol. 18, no. 6, pp. 1657$1675,1997$.

[9] T. Malas and L. Gürel, "Accelerating the multilevel fast multipole algorithm with the sparse-approximate-inverse (SAI) preconditioning," SIAM J. Sci. Comput., vol. 31, no. 3, pp. 1968-1984, 2009.

[10] M. Benzi, G. H. Golub, and J. Liesen, "Numerical solution of saddle point problems," Acta Numer, vol. 14, pp. 1-137, 2005.

[11] L. N. Trefethen and D. Bau, III, Numerical Linear Algebra. Philadelphia, USA: SIAM, 1997.

[12] C. Siefert and E. de Sturler, "Preconditioners for generalized saddlepoint problems," SIAM J. Numer. Anal., vol. 44, no. 3, pp. 1275-1296, 2006.

[13] G. H. Golub and C. F. van Loan, Matrix Computations. Johns Hopkins University Press, 1996

[14] Y. Saad, Iterative Methods for Sparse Linear Systems, 2nd ed. Philadelphia, USA: SIAM, 2003.

[15] T. Malas and L. Gürel, "Incomplete LU preconditioning with the multilevel fast multipole algorithm for electromagnetic scattering," SIAM J. Sci. Comput., vol. 29, no. 4, pp. 1476-1494, 2007.

[16] A. P. Pavacic, D. L. del Ro, J. R. Mosig, and G. V. Eleftheriades, "Threedimensional ray-tracing to model internal reflections in off-axis lens antennas," IEEE Trans. Antennas Propagat., vol. 54, pp. 604-612, 2006 